\title{
Instability in the Hotelling's Non-Price Spatial Competition Model
}

\author{
Yosuke Yasuda \\ National Graduate Institute for Policy Studies, Tokyo, Japan \\ Email: yyasuda@grips.ac.jp
}

Received April 13, 2013; revised May 13, 2013; accepted June 13, 2013

Copyright (C) 2013 Yosuke Yasuda. This is an open access article distributed under the Creative Commons Attribution License, which permits unrestricted use, distribution, and reproduction in any medium, provided the original work is properly cited.

\begin{abstract}
This note analyzes a slightly modified Hotelling model in which two firms are allowed to choose multiple store locations. Each firm can endogenously choose the number of stores while opening a store incurs a set-up cost. We show that the principle of minimum differentiation, i.e., both firms open a store each on the center, never holds when the set-up cost is decreasing in the number of stores. Under general cost functions that include non-linear and asymmetric set up costs, we characterize the conditions under which the principle holds. General payoff functions that are non-linear in the market share are also considered.
\end{abstract}

Keywords: Hotelling Model; Multiple Locations; The Principle of Minimization

\section{Introduction}

The spatial competition model initiated by Hotelling [1] is widely used in many fields such as business, economics, regional science, political economics, and so forth $^{1}$. The simplest version of the model, so-called the Hotelling model, considers the following situation: two firms simultaneously choose a location (of store) on a bounded line where potential customers are uniformly located. As we assume that each customer prefers the firm with closer location, this line structure captures heterogeneity of customers' preferences. The striking implication of the Hotelling model is that, trying to steal more customers from the rival, both firms end up choosing the center. This result, often referred as the principle of minimum differentiation, is employed to explain variety of concentrating phenomena, e.g., little product differentiation, agglomeration of shops, and similar target policies set by two political parties (the median voter theorem).

In this short note, we revisit the Hotelling model by incorporating the possibility that firms can choose multiple store locations. While there are many actual markets in which each firm sets up multiple shops, brands, faci-

\footnotetext{
${ }^{1}$ For the applications in the first three fields, see, for example, Eaton and Lipsey [2] and Gabszewicz and Thisse [3]. Hotelling [1] reinterpreted his model to explain the choice of political platforms in party competition, whose idea has been elaborated further by Downs [4] and others.
}

lities, etc, the literature on spatial competition with multiple stores is rather limited ${ }^{2}$. Teitz [6] first introduces multiple locations choice in the Hotelling model (under non-price competition), assuming that the number of stores each firm can operate is exogenously given ${ }^{3}$. Our model, in contrast, imposes no restriction on the firms' location choice: we allow each firm to endogenously choose the number of stores as well as their locations.

The most related analysis is given in a survey article by Gabszewicz and Thisse [10], which considers the essentially same model as ours. However, they focus only on the case of common and constant set-up cost ${ }^{4}$. Under such limited environment, they identify the cost parameter that supports pure strategy Nash equilibria. We extend their model by incorporating non-linear and asymmetric costs and derive the general condition under which the principle of minimum differentiation becomes valid.

One may argue that the assumption of single location choice imposed in the original Hoteling model could be merely a technical simplification and that considering

\footnotetext{
${ }^{2}$ For the related papers, see the literature survey described by Pal and Sarkar [5].

${ }^{3}$ There are a few papers that incorporate multiple locations choice into the spatial competition followed by other types of strategic decision, e.g., location-price (Martinez-Giralt and Neven [7]; Janssen et al. [8]), location-quantity (Pal and Sarkar [5]), and location-variety (Peng and Tabuchi [9]).

${ }^{4}$ I would like to thank an anonymous referee who mentioned this article.
} 
multiple store locations would not alter its main result, as long as the firms incur certain cost to set up each store. However, we show that agglomeration never occurs when there exists a common cost to set up each store, irrespective of its level.

Our characterization also shows that the range of cost parameters that support the principle of minimum differentiation turns out to be very small, even if we consider non-linear and asymmetric set up costs. For instance, the firms never agglomerate in the center if the set up cost is decreasing in the number of each firm's total stores. That is, whenever opening the second store costs less than the first (which seems to be satisfied in many actual situations), the principle becomes invalid. Our finding may call the caution against excessive use of the Hotelling model and its implication, especially when no institutional restriction prohibits players to choose multiple locations.

\section{Model}

On a line of length 1 , two firms $A$ and $B$ choose locations of their stores simultaneously. There is no (marginal) cost of production or operation, but the firms incur fixed cost to open stores. Customers are uniformly located on the line $[0,1]$. Each customer goes to the closest shop and buys exactly one unit of the good. If there are multiple stores with least distance from a customer, she randomly chooses each of them with equal probability. We abstract away pricing or producing decision by firms, and exclusively focus on their choice of store locations (product differentiations).

Unlike the simple Hotelling model in which every firm chooses only one location, we allow each firm to choose multiple locations. Let $X_{i}=\left\{x_{i}^{1}, x_{i}^{2}, \cdots, x_{i}^{N_{i}}\right\}$ denote firm i's strategy with $x_{i}^{1}<x_{i}^{2}<\cdots<x_{i}^{N_{i}}$ and $x_{i}^{n} \in[0,1]$ for $n=1,2, \cdots, N_{i}$, where $N_{i}$ represents the number of stores determined (endogenously) by firm $i=A, B$, i.e., $N_{i}=\left|X_{i}\right|$.

The payoff of each firm $i=A, B$, denoted by $u_{i}\left(X_{i}, X_{-i}\right)$, is written as

$$
u_{i}\left(X_{i}, X_{-i}\right)=s_{i}\left(X_{i}, X_{-i}\right)-c\left(N_{i}\right) \text {, }
$$

where $s_{i}$ is the share of the customers that firm $i$ obtains and $c$ is the total cost of opening $N_{i}$ stores. Note that our assumption of customers' behavior implies that $s_{A}+s_{B}=1$ always holds. Let $c(\cdot)$ be symmetric ${ }^{5}$ among firms and depend only on the number of stores. We also assume that $c(0)=0$ and $c(n)$ is non-decreasing in $n$.

To conclude the section, we provide the following re-

\footnotetext{
${ }^{5}$ Asymmetric costs with more general payoff functions than (1) will be
} analyzed in Section 4. marks that associate our model with the Hotelling model in which the firms can choose only one location each.

Remark 1. The Hotelling model can be considered as a special case of our model with $c(1)=0$ and $c(n)=\infty$ for any $n \geq 2$.

Remark 2. $X_{A}^{*}=X_{B}^{*}=\left\{\frac{1}{2}\right\}$ is a unique pure-strategy Nash equilibrium when $c(1)=0$ and $c(n)=\infty$ for any $n \geq 2$.

\section{When Firms Agglomerate in the Center}

In what follows, we consider whether the unique purestrategy Nash equilibrium of the Hotelling model, i.e., $X_{A}^{*}=X_{B}^{*}=\left\{\frac{1}{2}\right\}$, continues to be a Nash equilibrium in our model. To simplify the argument, let us first introduce the following lemma.

Lemma 1. If $c(1)>\frac{1}{2}$, there is no pure strategy Nash equilibrium such that each firm chooses at least one location.

Proof. Suppose that both firms choose at least one location each. Then, the sum of their payoffs necessarily becomes negative, since

$$
\begin{aligned}
& u_{A}+u_{B} \\
& =s_{A}-c\left(N_{A}\right)+s_{B}-c\left(N_{B}\right) \\
& =s_{A}+s_{B}-c\left(N_{A}\right)-c\left(N_{B}\right) \leq 1-2 c(1)<0 .
\end{aligned}
$$

This implies that at least one firm must incur negative payoff; such firm will be better off by not choosing any location.

Now we are ready to present our main results. The following theorem characterizes the condition under which $X_{A}^{*}=X_{B}^{*}=\left\{\frac{1}{2}\right\}$ becomes a Nash equilibrium.

Theorem 1. $X_{A}^{*}=X_{B}^{*}=\left\{\frac{1}{2}\right\}$ is a Nash equilibrium if and only if $c(2)-c(1) \geq \frac{1}{2} \geq c(1)$.

Proof. If part $(\Leftarrow)$ : Assume that both firms choose the center, $X_{A}=X_{B}=\left\{\frac{1}{2}\right\}$. Then,

$$
u_{i}\left(\frac{1}{2}, \frac{1}{2}\right)=s_{i}-c(1)=\frac{1}{2}-c(1) \geq 0
$$

for $i=A, B$. Since $X_{A}^{*}=X_{B}^{*}=\left\{\frac{1}{2}\right\}$ constitutes a Nash equilibrium in the simple Hotelling model, there is no profitable deviation with $N_{i}=0,1$. So, if a profitable deviation exists, the (deviating) firm must choose more 
than one location. However, for any $X_{i}$ with $N_{i} \geq 2$,

$$
u_{i}\left(X_{i}, \frac{1}{2}\right)=s_{i}-c\left(N_{i}\right) \leq 1-c(2)<\frac{1}{2}-c(1)=u_{i}\left(\frac{1}{2}, \frac{1}{2}\right),
$$

which implies that there is no profitable deviation. (Note that the first inequality comes from the fact that new profit becomes at most $1-c(2)$.)

Only if $\operatorname{part}(\Rightarrow)$ : We show the contrapositive, i.e., $X_{A}=X_{B}=\left\{\frac{1}{2}\right\}$ cannot be a Nash equilibrium if 1 ) $c(2)-c(1)<\frac{1}{2}$ or 2) $\frac{1}{2}<c(1)$. By Lemma 1 , no pure strategy Nash equilibrium exists when 2) $\frac{1}{2}<c(1)$. Therefore, it is enough to show that $X_{A}=X_{B}=\left\{\frac{1}{2}\right\}$ is not a Nash equilibrium when 1) $c(2)-c(1)<\frac{1}{2}$ (and $\frac{1}{2} \geq c(1)$ ). Then consider the following deviation by firm $A$ (from $X_{A}=X_{B}=\left\{\frac{1}{2}\right\}$ ), $\tilde{X}_{A}:=\left\{\frac{1}{2}-\epsilon, \frac{1}{2}+\epsilon\right\}$. Note that $A$ 's deviating profit becomes

$$
\begin{aligned}
u_{A}\left(\tilde{X}_{A}, \frac{1}{2}\right) & =1-\epsilon-c(2) \\
& =1-(c(2)-c(1))-c(1)-\epsilon>\frac{1}{2}-c(1)-\epsilon \\
& =u_{A}\left(\frac{1}{2}, \frac{1}{2}\right)-\epsilon .
\end{aligned}
$$

The inequality is derived by 1) $c(2)-c(1)<\frac{1}{2}$. Since $\epsilon$ could be arbitrary small, we obtain a profitable deviation.

Theorem 1 shows that the cost of opening a first store should be reasonably low (by Lemma 1) while the additional cost of opening a second store needs to be sufficiently high for the principle of minimization. The latter condition is needed since each firm has the following profitable deviation (from the agglomeration) when the set up cost of second store is low: choosing two locations such that the one store is slightly left and the other is slightly right to the center. This strategy gives the deviating firm almost all customers.

The theorem also implies the following corollary, which states that the principle becomes invalid when the set up cost of stores is non-increasing.

Corollary 1. If $2 c(1)>c(2)$, there is no Nash equilibrium such that $X_{A}^{*}=X_{B}^{*}=\left\{\frac{1}{2}\right\}$. When $2 c(1)=c(2)$, the above equilibrium exists if and only if $c(1)=\frac{1}{2}$.

Proof. By Theorem 1, $X_{A}^{*}=X_{B}^{*}=\left\{\frac{1}{2}\right\}$ cannot be a
Nash equilibrium if

$$
c(2)-c(1)<c(1) \Leftarrow c(2)<2 c(1),
$$

which establishes the first part. When $2 c(1)=c(2)$, we obtain (by Theorem 1)

$$
c(2)-c(1)=2 c(1)-c(1)=c(1) \geq \frac{1}{2} \geq c(1) .
$$

Clearly, the above inequalities hold if and only if

$$
c(1)=\frac{1}{2} \text {. }
$$

The first part of Corollary 1 shows that the principle of minimization never holds when the set up cost is decreasing. The second part shows that this impossibility result remains generically true even if the cost is constant. In short, strictly increasing set up cost is necessary to support the principle of minimization.

\section{Extension}

In the above analysis, we assume a simple payoff function defined in (1), which can be straightforwardly extended to more general cases. To illustrate this, let us incorporate payoff functions to be 1) asymmetric between firms and 2) non-linear in the market share. That is, for $i=A, B$,

$$
u_{i}\left(X_{i}, X_{-i}\right)=f_{i}\left(s_{i}\left(X_{i}, X_{-i}\right)\right)-c_{i}\left(N_{i}\right),
$$

where we assume that $f_{i}$ is a non-decreasing function of $s_{i}$ and continuous at $s_{i}=1$. These conditions are satisfied, for example, the Downs model which assumes

$$
f_{i}\left(s_{i}\right)=0 \text { for } 0 \leq s_{i}<\frac{1}{2}, f_{i}\left(s_{i}\right)=\frac{1}{2} \text { for } s_{i}=\frac{1}{2},
$$

and

$$
f_{i}\left(s_{i}\right)=1 \text { for } \frac{1}{2}<s_{i} \leq 1^{6} .
$$

Then, we obtain the following theorem.

Theorem 2. $X_{A}^{*}=X_{B}^{*}=\left\{\frac{1}{2}\right\}$ is a Nash equilibrium if and only if

$$
\forall i, \quad c_{i}(2)-c_{i}(1) \geq f_{i}(1)-f_{i}\left(\frac{1}{2}\right) \text { and } f_{i}\left(\frac{1}{2}\right) \geq c_{i}(1) .
$$

The proof is almost identical to that of Theorem 1, and thereby we skip it. Note that Theorem 1 is a special case

${ }^{6}$ This payoff specification initiated by Downs [4] is widely applied in political economics, which captures that the party winning a majority of votes gains control of the government. 
of Theorem 2 since the latter with $f_{i}(1)=1, f_{i}\left(\frac{1}{2}\right)=\frac{1}{2}$, and $c_{A}=C_{B}=C$ implies the former.

\section{Conclusion}

The note studies a modified Hotelling model in which each firm is allowed to choose multiple locations. Characterizing the condition under which the firms agglomerate in the center (Theorem 1), we show that the principle of minimum differentiation no longer holds unless the set up cost of opening a store is strictly increasing (Corollary 1). Our results may call the caution against directly applying the simple Hotelling model to the cases where no institutional restriction prohibits agents to choose multiple locations.

\section{Acknowledgements}

I would like to thank Michihiro Kandori, Hitoshi Matshushima, Noriaki Matsushima and Daisuke Oyama for helpful comments. All remaining errors are mine. This research was supported by Grant-in-Aid for Scientific Research (B) \#24330087 (2012-2016), the Ministry of Education, Culture, Sports, Science and Technology, Japan.

\section{REFERENCES}

[1] H. Hotelling, "Stability in Competition,” Economic Journal, Vol. 39, No. 153, 1929, pp. 41-57. doi: $10.2307 / 2224214$

[2] B. C. Eaton and R. G. Lipsey, "Product Differentiation," In: R. Schmalensee and R. D. Willig, Eds., Handbook of Industrial Organization, Volume I, Elsevier Science Pub- lishers, Amsterdam, 1989, pp. 723-768. doi:10.1016/S1573-448X(89)01015-0

[3] J. J. Gabszewicz and J.-F. Thisse, "Location,” In: R. J., Aumann and S. Hart, Eds., Handbook of Game Theory with Economic Applications, Volume I, Elsevier Science Publishers, 1992, pp. 281-304.

[4] A. Downs, "An Economic Theory of Political Action in a Democracy,” Journal of Political Economy, Vol. 65, No. 2, 1957, pp. 135-150. doi:10.1086/257897

[5] D. Pal and J. Sarkar, "Spatial Competition among MultiStore Firms," International Journal of Industrial Organization, Vol. 20, No. 2, 2002, pp. 163-190. doi:10.1016/S0167-7187(00)00080-1

[6] M. B. Teitz, "Locational Strategies for Competitive Systems,” Journal of Regional Science, Vol. 8, No. 2, 1968, pp. 135-148. doi:10.1111/j.1467-9787.1968.tb01319.x

[7] X. Martinez-Giralt and D. J. Neven, "Can Price Competition Dominate Market Segmentation?” Journal of Industrial Economics, Vol. 36, No. 4, 1988, pp. 431-442. doi:10.2307/2098448

[8] M. C. W. Janssen, V. A. Karamychev and P. Van Reeven, "Multi-Store Competition: Market Segmentation or Interlacing?” Regional Science and Urban Economics, Vol. 35, No. 6, 2005, pp. 700-714. doi:10.1016/j.regsciurbeco.2004.12.002

[9] S.-K. Peng and T. Tabuchi, "Spatial Competition in Variety and Number of Stores," Journal of Economics and Management Strategy, Vol. 16, No. 1, 2007, pp. 227-250. doi:10.1111/j.1530-9134.2007.00138.x

[10] J. J. Gabszewicz and J.-F. Thisse, "Spatial Competition and The Location of Firms," In: J. J. Gabszewicz, J.-F., Thisse, M. Fujita and U. Schweizer, Eds., Location Theory, Harwood Academic Publishers GmbH, Newark, 1986, pp. 1-71. 